\title{
Cervicobrachial neuralgia revealing a meningioma
}

\section{Keywords: Cervico-brachial neuralgia, Spinal MRI, Surgery Clinical images}

This is a 52-year-old patient who has been hospitalized for a straight-necked cervico-brachial neuralgia $\mathrm{C} 7$ of mixed type, evolving for a year, not improved by medical treatment. The examination showed a supple but painful cervical spine to movements of laterality and rotation, with abolished reflexes to the upper right limb without sensory-motor deficit. Biology did not reveal any inflammatory syndrome. The radiographs of the cervical spine showed a C4-C5 vertebral block. Spinal MRI revealed the presence of an intra-dural, extra-medullary expansive process, anterior seat located on the left opposite D1, intermediate signal on the T1 sequences (Panel A) and hypersignal on the T2 sequences (Panel B), enhanced homogeneously after gadolinium injection, measuring $2 \times 1 \mathrm{~cm}$, exerting a mass effect on the spinal cord without signal anomaly at the marrow, compatible with a meningioma (Panel C). A surgical treatment involving a D1D2 laminectomy with complete removal of the tumor was performed. The histology of the surgical specimen concluded with a meningioma. Meningioma is one of the intra-dural and extra-medullary tumors. It can be at the origin of a tenacious NCB. The pain is intense, while cervical stiffness may be lacking. Spinal MRI shows the tumor and its repercussion on the marrow. The treatment of meningioma is surgical (Figure 1).

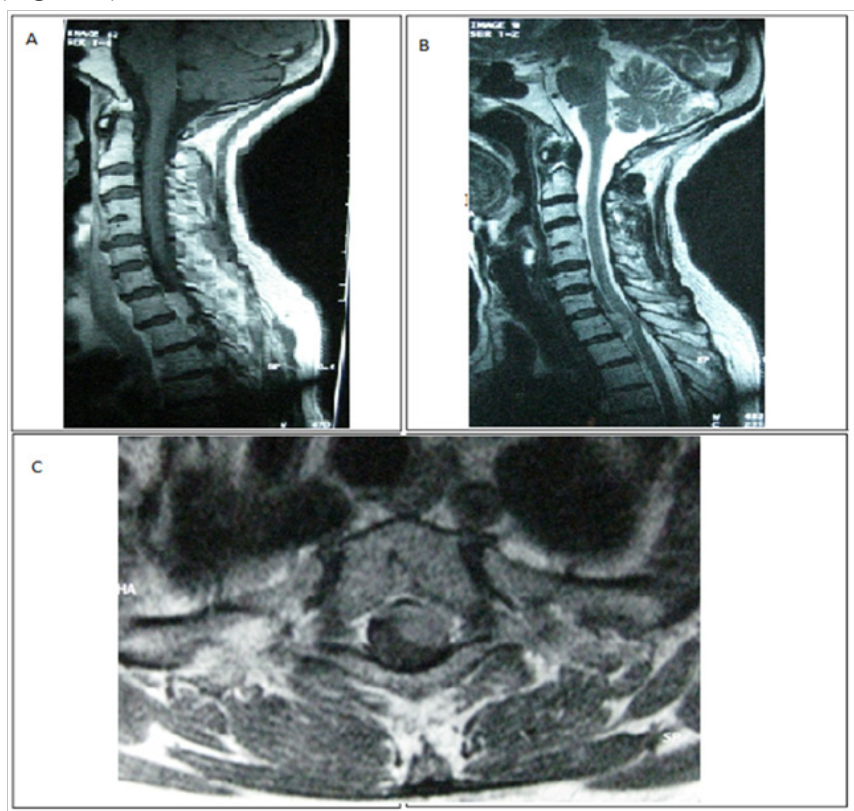

Figure I Spinal MRI: Intra-dural, extramedullary expansive process of anterior seat located on the left opposite DI, intermediate signal on the TI sequences (A) and hypersignal on the T2 sequences (B), enhancing homogeneously after injection of gadolinium, measuring $2 x \mathrm{l} \mathrm{cm}$, exerting a mass effect on the medullary cord without signal abnormality at the marrow (C).
Volume 7 Issue 3 - 2017

Zeineb Alaya,' Lassad Hassini, ${ }^{2}$ Walid Osman, ${ }^{2}$ Nader Naouar, ${ }^{2}$ Mohamed Laziz Ben Ayèche, ${ }^{2}$ Elyès Bouajina'

'Department of Rheumatology, Farhat Hached Hospital, Faculty of Medicine of Sousse, Tunisia

${ }^{2}$ Department of Orthopaedics, Sahloul Hospital, Faculty of Medicine of Sousse, Tunisia

Correspondence: Zeineb Alaya, Department of Rheumatology, Farhat Hached Hospital, Faculty of medicine of Sousse, Ibn el Jazzar Street, 4000 Sousse, Tunisia, Tel 21698375975, Email zeineb_a@hotmail.fr

Received: February II, 2017| Published: February 15, 2017

\section{Acknowledgments}

None.

\section{Conflicts of interest}

None. 